Cahiers « Mondes anciens »

ANCIENS

Histoire et anthropologie des mondes anciens

$7 \mid 2015$

Marchands romains au long cours

\title{
Les maisons des négociants italiens à Délos : structuration de l'espace domestique dans une société en mouvement
}

The Houses of Italian Merchants on Delos: the Structuring of Domestic Space in a Society in Motion

\section{Mantha Zarmakoupi}

\section{OpenEdition \\ Journals}

Édition électronique

URL : http://journals.openedition.org/mondesanciens/1588

DOI : 10.4000/mondesanciens. 1588

ISSN : 2107-0199

Éditeur

UMR 8210 Anthropologie et Histoire des Mondes Antiques

Référence électronique

Mantha Zarmakoupi, «Les maisons des négociants italiens à Délos : structuration de l'espace

domestique dans une société en mouvement », Cahiers " Mondes anciens » [En ligne], 7| 2015, mis en ligne le 21 décembre 2015, consulté le 01 mai 2019. URL : http://journals.openedition.org/ mondesanciens/1588; DOI : 10.4000/mondesanciens.1588

Ce document a été généré automatiquement le 1 mai 2019.

Les Cahiers «Mondes Anciens » sont mis à disposition selon les termes de la licence Creative Commons Attribution - Pas d'Utilisation Commerciale - Pas de Modification 4.0 International. 


\section{Les maisons des négociants italiens à Délos : structuration de l'espace domestique dans une société en mouvement}

The Houses of Italian Merchants on Delos: the Structuring of Domestic Space in a Society in Motion

Mantha Zarmakoupi

1 La prospérité du commerce à Délos résulta de la décision du Sénat romain de créer un port franc sous la souveraineté athénienne, en 167 avant notre ère ${ }^{1}$. Selon les sources littéraires (Strabon, XIV, 5, 2; Pline, Naturalis Historia, 34, 9; Pausanias, III, 23, 3-6; et Lucilius [Paulus, ex Festo 88, 4]), des esclaves et des produits de luxe (tels que les parfums, épices, verreries ou tissus) provenant du Moyen et Extrême-Orient étaient échangés à Délos $^{2}$, tandis que certains produits étaient fabriqués sur place ${ }^{3}$.

2 Avec une population 1500 à 2000 d'habitants environ à l'époque de l'indépendance, Délos atteignit environ 15000 d'habitants durant la période de la seconde domination athénienne. Il a été suggéré que l'île abrita une population de 20 à 30000 habitants à son apogée ${ }^{4}$. Cependant, il n'en existe aucune preuve solide. Les inscriptions témoignent de la présence de 1200 citoyens et d'une population d'environ 6000 habitants au début du I ${ }^{\text {er }}$ siècle avant notre ère. Pendant cette période, l'île se caractérisa par son cosmopolitisme. La majorité de ses nouveaux habitants venaient de la péninsule italienne et de Méditerranée orientale. Alors que certains marchands venaient de villes proches de l'Ouest et du Sud de l'Asie Mineure, une grande partie d'entre eux provenait de terres lointaines, notamment de Gadara, d'Héliopolis, d'Arabie, de Nabatène et de Gerra du golfe Persique $^{5}$. La majorité des habitants de l'île était cependant des Italiens et des Romains ${ }^{6}$.

Bien que la société locale soit diversifiée, l'architecture des maisons - du moins des rezde-chaussée qui sont préservés - était assez uniforme. Elle procédait de ce qu'on a appelé 
une koinè architecturale. Contrairement aux tendances ultérieures à la "romanisation » des territoires où les Romains se sont installés, on note une adaptation totale aux techniques de construction locales, même si les inscriptions, les peintures et les sculptures attestent l'identité religieuse et ethnique des propriétaires ${ }^{7}$. Des études récentes ont indiqué que le contact des Romains avec les cultures locales a entraîné une pluralité d'expressions culturelles hybrides, qui défient la généralisation que suppose le terme de "romanisation " ${ }^{8}$. Même si la "romanisation " reste un outil méthodologique indispensable et une des approches nécessaires de l'histoire de Rome, il est essentiel de mieux comprendre les phases des échanges culturels que le contact de Rome avec les sociétés provinciales, et plus particulièrement la Grèce, a entraînég. La Grèce se situe aux antipodes de la romanisation, car le processus "d'hellénisation» était une étape essentielle de la «romanisation». L'identité romaine a pris sa forme pendant un processus d'assimilation, imitation, appropriation et création en opposition avec la culture grecque ${ }^{10}$. Le cas de Délos, un des lieux du premier échange entre la culture romaine et la culture grecque, peut contribuer à élucider ce processus.

4 À Délos, les Romains - comme tous les étrangers - ont adopté le langage architectural local et se sont mis à aménager leurs maisons et les sièges de leurs associations religieuses autour d'une cour, tandis que les détails de la décoration, comme des statues et des peintures murales, ainsi que les inscriptions, indiquaient leur identité propre ${ }^{11}$. La Maison de Cléopâtre (GD 119)12 qui fut occupée par un couple provenant d'Athènes (Dioskourides et Cléopâtre du dème de Myrrhinonte [ID 1987]) ${ }^{13}$ n'est pas différente de la Maison du Trident (GD 118), peut-être construite par un Syrien, comme l'indiquent les protomés des taureaux de Hadad et les lions d'Atargatis présents dans le péristyle rhodien de la maison ${ }^{14}$, ou de la Maison des dauphins (GD 111) construite très probablement par un Phénicien, comme le suggère la signature de la mosaïque de la cour (Asklepiades d'Arados) et la présence du symbole de Tanit sur la mosaïque ${ }^{15}$, et finalement des maisons des Italiens comportant des peintures murales à caractère religieux, mettant en scène le culte romain de Lares compitales ${ }^{16}$. Tout aussi homogène dans leur architecture sont les sièges des associations religieuses, par exemple celle de l'association des Poseidoniastes de Bérytos $(\mathrm{GD} 57)^{17}$.

5 Peut-on repérer les «marchands romains » dans les données archéologiques que Délos nous a fournies? Les inscriptions révèlent que la plupart des Italiens présents à Délos étaient issus de Rome, du Latium et de Campanie, et que certains venaient d'une dizaine de cités grecques d'Italie méridionale ${ }^{18}$. Même si leur origine géographique paraît cohérente, leurs statuts juridiques n'étaient pas uniformes: les citoyens romains se mêlaient aux Latins et aux alliés.

6 À partir de la façon dont les Italiens et les Romains sont nommés dans les inscriptions, il semble que $\mathrm{P} \omega \mu \alpha \tilde{i} o \zeta$ avait un sens très large ${ }^{19}$. Jean Hatzfeld puis Claire Hasenohr ont démontré que, dans certaines inscriptions, $\mathrm{P} \omega \mu \alpha$ ĩos pouvait désigner un citoyen romain, mais il y en a d'autres où il était appliqué à quiconque venait d'Italie. Certains Italiens se nommaient eux-mêmes ' $\mathrm{P} \omega$ uxĩos, parce qu'il était ainsi plus aisé de se faire comprendre. Il semble que des intérêts professionnels aient uni les Romains et les Italiens de Délos sous le label de "Romains", non pas dans le sens de citoyens romains, mais comme un groupe ethnique. Celui-ci se distinguait par son origine géographique commune (l'Italie) et son identité s'est définie par contact et juxtaposition avec les autres groupes ethniques de marchands de Délos, d'autre origine géographique (de Beyrouth, par exemple). Considérés comme des 'P $\omega \mu \alpha$ ĩor par les autres habitants de l'île, les Italiens ont repris à 
leur compte une certaine conception de la romanité, qui leur a été utile du point de vue politique : Romains et Italiens étaient unis par une origine géographique et des intérêts professionnels communs ${ }^{20}$. Comme Claire Hasenohr l'a proposé, on doit plutôt parler d'un " groupe ethnique ${ }^{21}$. De ce fait, j'ai choisi d'employer le terme " négociants italiens » au lieu de « négociants romains » pour étudier les maisons de ce groupe.

7 L'étude de l'architecture domestique de Délos est déterminée par la nature des données existantes. Celles-ci proviennent de fouilles effectuées à la fin du XIX ${ }^{e}$ siècle et au début du $\mathrm{xx}^{\mathrm{e}}$ siècle. La stratigraphie n'a pas été relevée et les objets n'ont pas été enregistrés en même temps que leur contexte archéologique, comme dans toutes les fouilles de cette époque. La seule exception est celle du quartier Nord, fouillé dans les années 1960 et 1970 par Philippe Bruneau et Gérard Siebert ${ }^{22}$. C'est pour cette raison que depuis le début du XX e siècle, l'analyse typologique de l'architecture du Quartier du Théâtre donne des informations sur l'organisation et le fonctionnement des maisons, mais pas sur leur développement ni sur les changements d'usage de leurs espaces.

8 L'analyse typologique des maisons effectuée durant le siècle dernier a fourni des résultats importants, mais a aussi débouché sur des impasses à cause de la conceptualisation schématique de l'espace domestique. Les pièces des maisons déliennes sont groupées autour d'une cour et la présence d'un péristyle semble être un élément caractéristique. L'analyse monumentale consacrée par Joseph Chamonard aux maisons du Quartier du Théâtre a fourni une typologie des salles et maisons où les concepts d'oecus maior et d' oecus minor définissent l'organisation des salles de réception et de représentation ${ }^{23}$. Selon ce schéma, l'oecus maior, en relation directe avec la cour, correspond à une grande pièce luxueuse, donnant accès à une ou deux petites salles, appelées oeci minores. Cet ensemble de salles était probablement utilisé pour de nombreuses activités de la vie quotidienne, et surtout pour dîner et se divertir. Ce schéma typologique répond au goût du luxe qui caractérisait le monde hellénistique, comme le montrent les exemples de Rhodes, Kos et Thasos $^{24}$.

9 Ces concepts sont applicables à une grande partie des maisons déliennes, en particulier aux grandes. En revanche, ce schéma typologique n'est pas adéquat pour analyser les maisons transformées pour accueillir des ateliers ou d'autres activités commerciales et où l'importance des salles de représentation a progressivement diminué. L'insuffisance de ce schéma a été démontrée par Monika Trümper. En utilisant la distinction entre " public » et "privé», tout en faisant une analyse exhaustive des maisons déliennes, cette chercheuse a montré leur pluralité et leur polyvalence ${ }^{25}$. Son étude de la fonction des salles, de l'organisation et du développement des maisons a amélioré notre compréhension des espaces domestiques et de leurs évolutions. Plus récemment, en reprenant le discours sur l'espace public et privé dans son étude sur l'habitat ancien, Lisa Nevett a utilisé la méthode de la syntaxe spatiale («Space Syntax Analysis») pour analyser les maisons déliennes ${ }^{26}$. Elle a démontré le postulat initial de Joseph Chamonard ${ }^{27}$ selon lequel, à de rares exceptions, l'espace des maisons déliennes était peu visible des passants. Grâce aux statistiques, Lisa Nevett a également montré, comme l'avait proposé Philippe Bruneau ${ }^{28}$, que les maisons déliennes n'ont pas été influencées par les maisons italiennes, exception faite de quelques exemples célèbres, comme la Maison du Trident (GD 118). Malgré les nouvelles perspectives fournies par les études de Monika Trümper et de Lisa Nevett, la particularité de l'économie et de la société déliennes n'a pas été prise en compte dans l'analyse du développement de l'habitat. Mon étude a pour but de compléter 
leurs approches et de lier certaines particularités de l'architecture à la nature dynamique de l'économie de Délos à la fin de l'époque hellénistique.

Fig. 1. Quartier du Stade, Maison IC, autel à droite de l'entrée de la maison

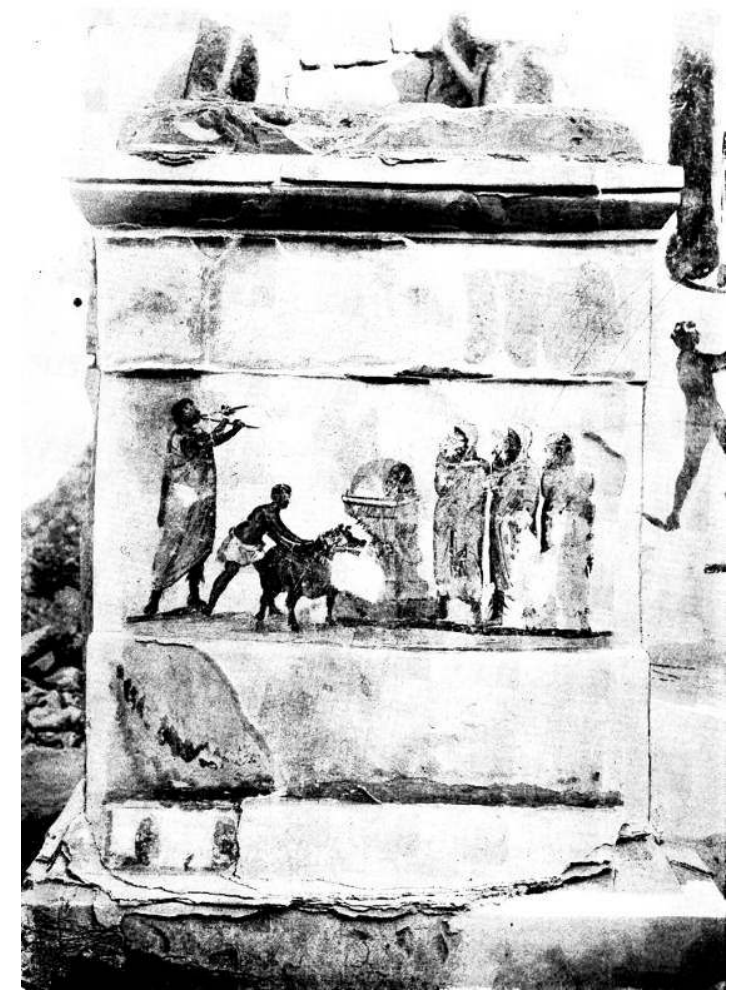

Plassart A. (1916), «Fouilles de Délos, exécutées aux frais de M. le Duc de Loubat (1912-1913). Quartier des habitations privées à l'est du Stade (pl. V-VII)», BCH 40, p. 44.

10 Même si l'architecture et le décor des maisons déliennes ne reflètent pas l'origine de leurs occupants, les maisons italiennes se distinguent incontestablement, plus que celles de tout autre groupe, par les peintures liturgiques du culte de Lares Compitales qui ornaient les murs et les autels installés aux portes des maisons. Parmi les maisons qui ont été fouillées à Délos, 18 comportent des peintures liées au culte de Lares ${ }^{29}$. Les individus qui y sont représentées sont clairement italiens : ils portent des toges (et parfois des praetextae) et des calcei à leurs pieds. En outre, ils sacrifient ritu romano avec la tête voilée (voir par exemple la représentation de l'autel à droite de l'entrée de la Maison IC, dans le Quartier du Stade [GD 79], fig. 1). Les personnes citées par les inscriptions sont des esclaves et des affranchis, principalement de Méditerranée orientale. L'interprétation la plus plausible est donc que les esclaves et les affranchis des inscriptions étaient les serviteurs de familles italiennes. Il est probable que certains liberti géraient les affaires de leurs patrons. Il est possible que les Compitalia aient été célébrées par les premiers Italiens installés à Délos, car elles permettaient la réunion d'un groupe socialement hétérogène : les esclaves et les affranchis d'origine grecque et orientale, qui représentaient des maîtres restés en Italie. L'adoption de ce culte romain leur permettait de s'intégrer au groupe ethnique italien ${ }^{30}$.

11 L'étude de ces peintures, que Claire Hasenohr a rapprochées des monuments inscrits par le collège des Compétaliastes, montre que les Compitalia étaient célébrés dans un contexte à la fois privé (par des sacrifices aux portes des maisons) et public (par un sacrifice 
commun des Italici dans le sanctuaire des Lares) ${ }^{31}$. La fête des Compitalia était célébrée en l'honneur de divinités protectrices des rues et des carrefours, ce qui renforçait les liens de voisinage. L'omniprésence des peintures liturgiques de Lares compitales, qui ornaient murs et autels aux portes des maisons, est frappante dans le paysage urbain de Délos. Ces peintures soulignaient non seulement l'identité du groupe ethnique, mais renforçaient aussi ses liens communautaires en se référant à des monuments publics propres, qui marquaient le paysage délien : l'Agora des Compétaliastes (GD 2) et l'Agora des Italiens (GD 52) 32 .

12 Cependant, si les façades des maisons de négociants italiens renforçaient l'identité ethnique du groupe en faisant référence au culte des Lares compitales, les intérieurs furent façonnés pour satisfaire des besoins économiques et en même temps pour se conformer à un besoin de souligner un statut social.

13 Comme toutes les maisons déliennes de cette période, les maisons des négociants italiens ont été modifiées plusieurs fois, afin d'accueillir les besoins de leurs occupants. Le rez-dechaussée des maisons déliennes était traditionnellement organisé autour d'une cour, avec des salles de réception et de représentation autour d'elle, selon le schéma d'oecus maior et oecus minor expliqué auparavant. Même si cette organisation initiale fut maintenue dans plusieurs maisons, certains rez-de-chaussée furent modifiés pour abriter d'autres activités, en particulier économiques, dans des ateliers ou des lieux de stockage ${ }^{33}$. Souvent, le réaménagement du rez-de-chaussée pour accueillir des activités économiques a été combiné avec la présence d'un étage supérieur, où la luxueuse résidence du propriétaire était déplacée. Les maisons où l'on note l'association d'activités économiques au rez-de-chaussée à un étage luxueux appartenaient aux négociants italiens. Cette conjonction ne résulte certainement pas du hasard. Les deux réaménagements servirent, d'une part, les besoins économiques des occupants et, d'autre part, leur besoin de souligner leur statut social. 
Fig. 2. Quartier Nord, Maison de Sceaux, plan.

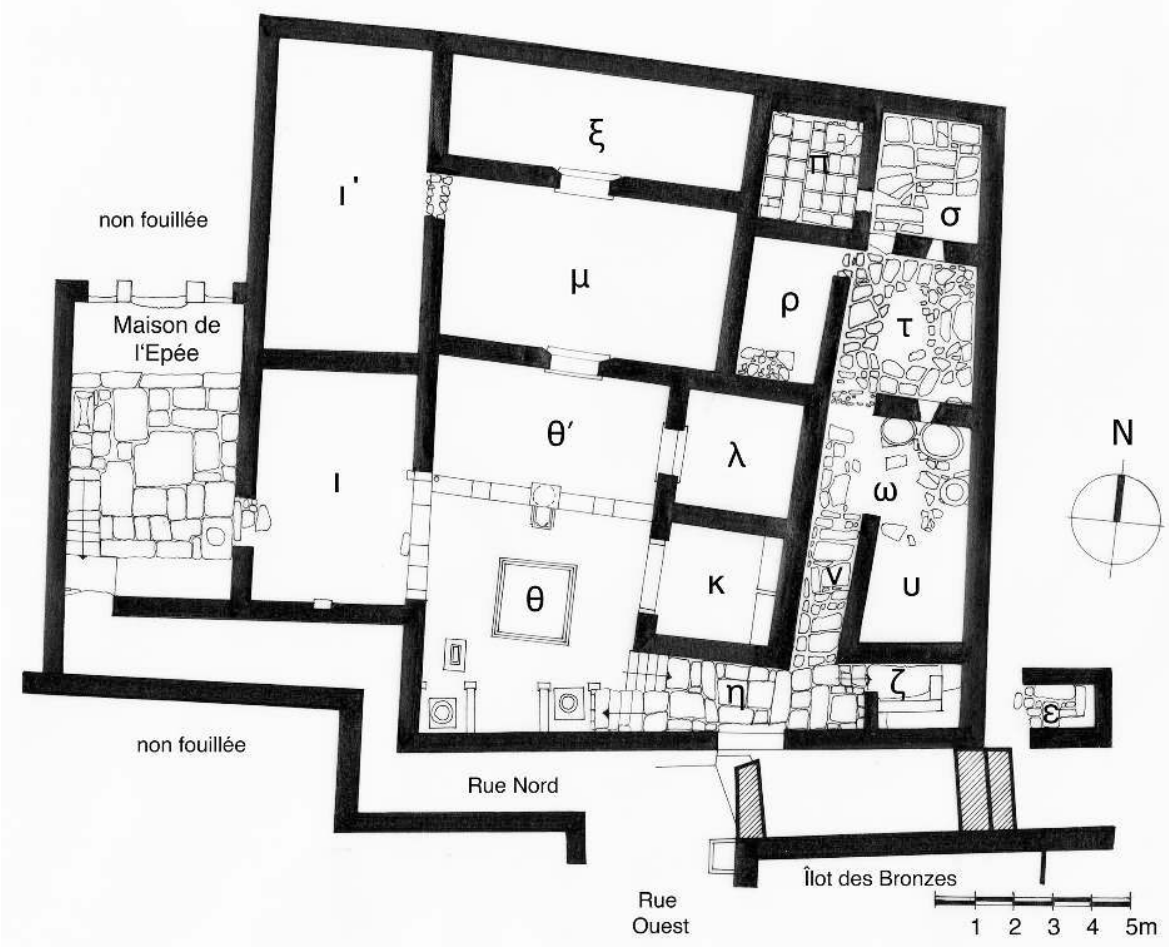

Mantha Zarmakoupi, d'après Siebert 2001, plan IV.

Fig. 3. Quartier du Stade, Maisons IC and ID, première phase, plan.

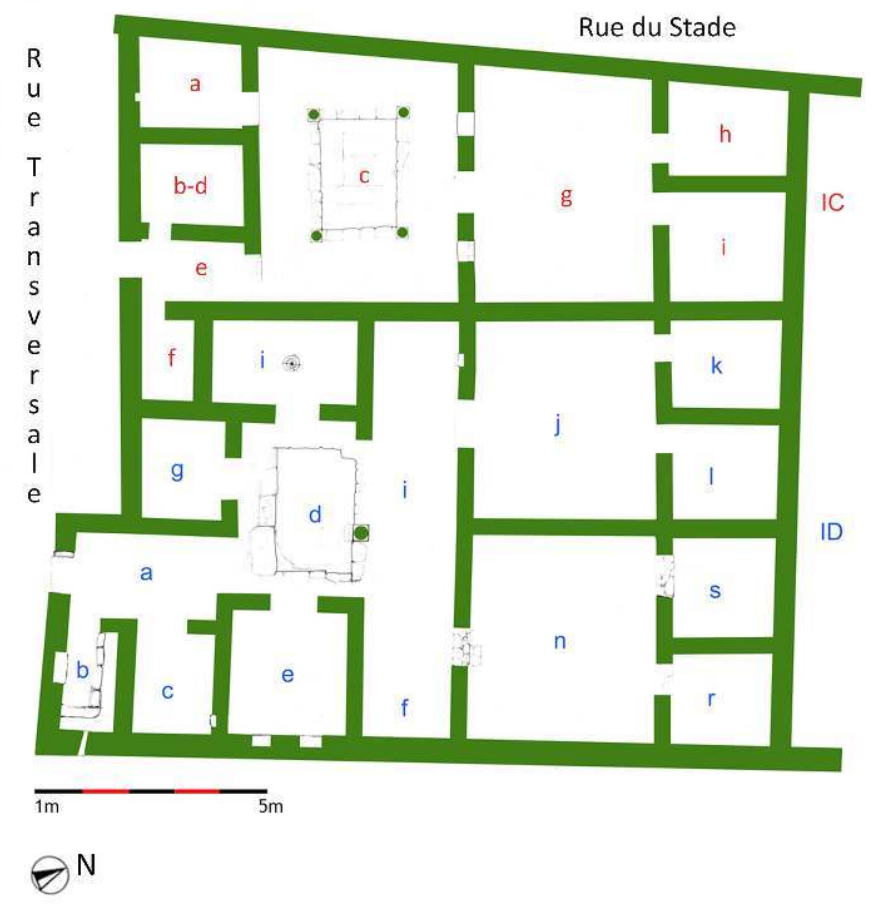

Quartier du Stade, îlot I, maisons IC et ID, phase 1 
14 Je concentrerai mon enquête sur trois maisons : la Maison des Sceaux (GD 59 D) ${ }^{34}$ dans le Quartier Nord, la maison de Quintus Tullius, ou Maison IC $^{35}$, et la Maison ID $^{36}$, dans le Quartier du Stade (GD 79). La Maison des Sceaux (fig. 2), dans le Quartier Nord, est une célèbre demeure, où un lot de 16000 sceaux a été mis au jour et a donné son nom à l'édifice (128/7 - 69 avant notre ère). Un grand nombre de ces objets porte les noms de familles italiennes connues à Délos ${ }^{37}$. Deux bustes "véristes ", qui venaient de l'étage supérieur, ont été interprétés comme la représentation des propriétaires. Il s'agissait de financiers ou de négociants italiens - peut-être des célèbres financiers L. Aufidius Bassus père et fils (maior et minor) ${ }^{38}$. La Maison ID, dans le Quartier du Stade (fig. 3), présente des peintures liturgiques à son entrée. La Maison IC (fig. 3), sise dans le même quartier, est l'une des trois maisons où est conservée, outre l'indication de Lares compitales à l'entrée (fig. 1), une inscription donnant le nom du propriétaire romain: Quintus Tullius ( $Q$. Tullius Q. f., ID 1802) ${ }^{39}$. Les trois maisons furent modifiées et même remodelées. Dans tous les cas, le rez-de-chaussée fut réorganisé, selon moi, pour accueillir des activités économiques, tandis que les salles de réception et de représentation furent aménagées à l'étage. Dans deux maisons sur trois - la Maison des Sceaux et la Maison de Quintus Tullius - l'étage fut ajouté dans un second temps, probablement au moment où les modifications au rez-de-chaussée eurent lieu, pour accueillir les salles de réception et de représentation ${ }^{40}$.

Dans le cas de la Maison des Sceaux, où les données archéologiques ont été conservées par un incendie, on a la preuve que la réorganisation du rez-de-chaussée a fait place à un atelier, à une petite entreprise et à des espaces de stockage. La partie orientale a accueilli un magasin et un atelier pour le traitement du grain, la production du vin et peut-être d'huile d'olive. Ce secteur avait un accès presque indépendant de l'entrée principale de la maison. Au même moment, dans la partie occidentale, la maison fut agrandie. Un groupe de salles, qui aurait pu fonctionner individuellement et être loué à une entreprise ou comme chambres à coucher, y fut formé, tandis que les salles disposées autour de la cour furent utilisées comme des espaces de stockage. Dans les deux autres cas, la similitude entre l'organisation architecturale du rez-de-chaussée et la dernière phase du rez-dechaussée de la Maison des Sceaux, d'une part, et la concentration de tessons des amphores ou d'autres trouvailles dans des salles du rez-de-chaussée, d'autre part, suggèrent la présence d'activités économiques ${ }^{41}$. 
Fig. 4. Quartier du Stade, Maisons IC et ID, deuxième phase, plan.

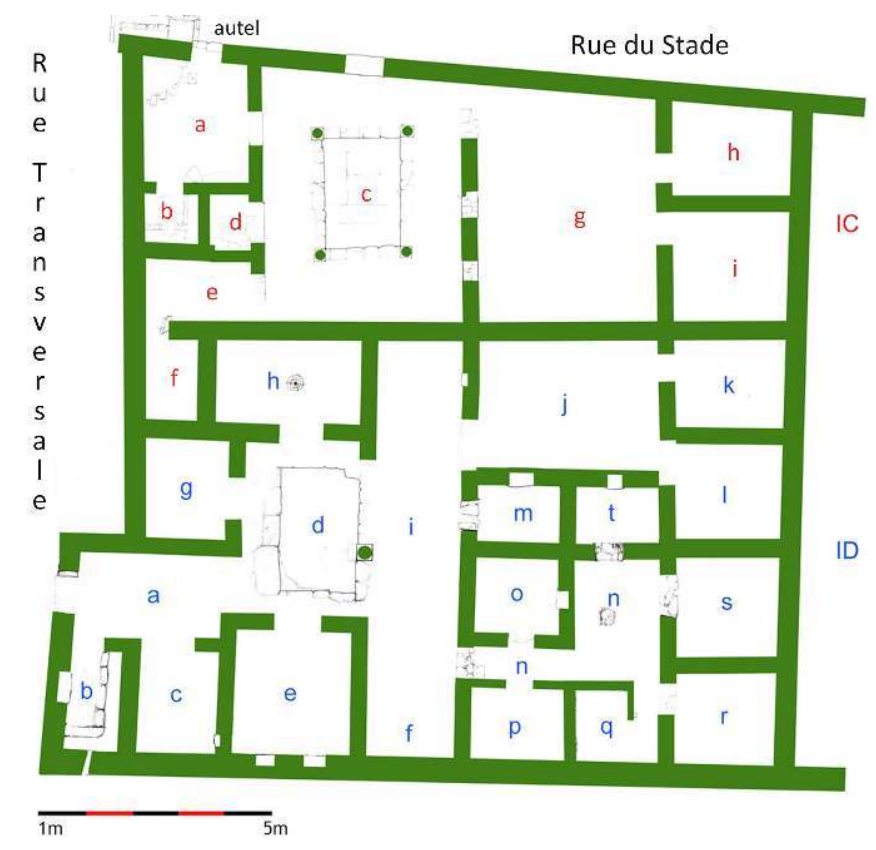<smiles>[CH]</smiles>

Quartier du Stade, îlot I, maisons IC et ID, phase 2

Mantha Zarmakoupi

Ainsi, au rez-de-chaussée de la Maison ID, deux grandes pièces ont été divisées en plusieurs petites salles (cf. fig. 4). Elles étaient accessibles depuis la cour et auraient pu fonctionner indépendamment du reste de la maison, comme les groupes de salles de la Maison des Sceaux. Une pièce (k) a livré les preuves archéologiques de son utilisation comme espace de stockage : de nombreuses amphores et un dépôt de tuiles y ont été trouvés ${ }^{42}$. La phase ultérieure du rez-de-chaussée de la Maison IC, dans le Quartier du Stade, présente des modifications comparables (fig. 3 et 4$)^{43}$. Avec l'addition du deuxième étage, l'entrée de la maison fut aménagée du côté ouest et les salles au nord et au sud-est de la cour furent réservées pour du stockage, tandis que l'étage accueillit les salles de représentation et de réception. La base et la statue de Quintus Tullius y étaient installées. Dans ces trois maisons, les modifications effectuées ont créé des groupes de pièces qui pouvaient fonctionner indépendamment, pour accueillir des ateliers, des entreprises, des lieux de stockage ou même des chambres à coucher. Il est aussi possible que ces pièces aient été louées ${ }^{44}$.

17 Les maisons à étages luxueux ne sont pas spécifiques aux maisons des Italiens ${ }^{45}$, mais la combinaison d'un étage luxueux avec un rez-de-chaussée accueillant des activités économiques est corroborée seulement pour les trois maisons qui ont été analysées ici et qui appartenaient aux Italiens. En outre, deux autres maisons occupées par des Italiens présentent un étage luxueux avec des modifications au rez-de-chaussée (comportant des chambres pouvant être utilisés séparément et être loués) : Maison VI H du Quartier du Théâtre et Habitation II de l'îlot des bijoux. Les maisons qui peuvent être identifiées comme occupées par des Italiens s'élèvent à vingt: dix-sept sont attestées par des peintures liturgiques ${ }^{46}$, une est attestée par des peintures liturgiques et une inscription 
(Maison IC du Quartier du Stade ; ID 1802 ${ }^{47}$ ), une autre par une inscription (Maison E situé à l'Est du peribolos oriental du sanctuaire d'Apollon [GD 82] ; ID 2378 ${ }^{48}$ ) et une dernière par des sceaux (Maison des Sceaux). Parmi les dix-huit maisons ornées de peintures liturgiques liées aux Lares compitales, cinq n'ont pas été fouillées. Donc sur quinze maisons d'Italiens fouillées, cinq (soit le tiers) combinent un étage luxueux et un rez-dechaussée accueillant des activités économiques.

Alors que les façades des maisons des négociants italiens ont renforcé l'identité du groupe ethnique en se référant au culte des Lares compitales, les intérieurs ont été modifiés pour répondre à des besoins précis. Cette conjonction n'est certainement pas due au hasard et révèle un élément italien dans l'organisation de ces maisons. Les négociants italiens ont ajouté des salles de réception et de représentation au-dessus du rez-de-chaussée, qui répondait aux besoins de leurs activités économiques croissantes. Ils s'enrichirent tout en affichant leurs profits aux yeux de leurs clients. Cette combinaison est bien connue dans les maisons et villas romaines d'Italie, à une date un peu plus tardive, proche de la moitié $\mathrm{du} \mathrm{I}^{\mathrm{er}}$ siècle avant notre ère ${ }^{49}$. Dans les villas romaines, des parties luxueuses sont associées à des parties qui servaient à l'agriculture. Comme Nicholas Purcell l'a montré, les Romains ont utilisé l'agriculture et l'élégance comme formes alternatives pour se révéler dans leur milieu social (" alternative forms of display $"^{50}$ ). C'est dans ce contexte qu'il faut comprendre cette nouvelle organisation des maisons de négociants italiens à Délos. Tandis que les activités du rez-de-chaussée servaient leurs besoins économiques, la combinaison de l'étage luxueux et du rez-de-chaussée servait leurs aspirations sociales.

Dans la Maison IC du Quartier du Stade, analysée auparavant, une inscription donne le nom du propriétaire romain, Quintus Tullius (ID 1802) ) $^{51}$. L'inscription est bilingue, en grec et en latin, et commémore l'érection d'une statue par trois affranchis, Héracléon, Alexandros et Aristarchos, à leur patron (Q. Tullius Q. f. $)^{52}$. Le patron et son affranchi Héracléon sont connus par d'autres inscriptions. Quintus Tullius fut Apolloniaste (prêtre d'Apollon) en 125 avant notre ère (ID 1730) ${ }^{53}$. L'inscription de la Maison IC est plus tardive, comme Plassart l'a souligné, étant donné que Héracléon apparait encore comme esclave dans une dédicace de Compétaliastes datée en 97/96 avant notre ère (ID 1761) ${ }^{54}$. L'inscription se lit ainsi :

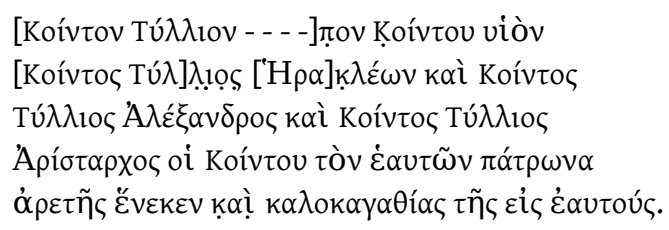

[(À) Quintus Tullius ---]pus fils de Quintus, Quintus Tullius Héracléon, Quintus Tullius Alexandros et Quintus Tullius Aristarchos (ont dédié cette statue) à leur patron, honorant sa vertu et les qualités dont il a fait preuve envers eux.

[Q. Tullium Q.f - - - pum]

Q. Tullius Q. 1. A[ristarchus]

Q. Tullius Q.1. Ale[xander]

Q. Tullius Q. 1. He[racle]o [p]atro[num]

suom honoris et be[nef]ici cau[sa]

(Pour) Quintus Tullius fils de Quintus, Quintus Tullius Aristarchos, Quintus Tullius Alexandros et Quintus Tullius Héracléon (ont dédié cette statue) à leur patron, pour sa vertu et les qualités dont il a fait preuve envers eux.

Les affranchis grecs de Quintus Tullius ont dédié la statue avec des inscriptions en grec et en latin. L'utilisation de la langue grecque par les affranchis indique la volonté de 
s'intégrer linguistiquement à la société délienne au nom de leur patron ${ }^{55}$. L'usage du latin, d'un autre côté, montre leur respect à l'égard de leur patron. Il est intéressant de noter que l'inscription grecque cite Héracléon en premier et Aristarchos en troisième, tandis que le latin inverse les positions d'Aristarchos et d'Héracléon. Il est possible que sur l'inscription latine, les affranchis aient tenu à souligner leur relation par rapport à leur patron, tandis que dans celle en grec, ils aient tenu à souligner leurs propres sentiments, comme Touloumakos l'a proposé56 ${ }^{5}$. Il est également possible que la différence ait visé à indiquer leurs relations différentes vis-à-vis des affaires du patron. Cette variation est de toute façon intéressante à souligner car elle indique que la relation entre les affranchis et le patron n'était pas sans ambiguïté.

Des études récentes ont montré que la fonction des inscriptions bilingues était de projeter une sorte d'identité collective des negotiatores présents à Délos et de construire l'image d'entreprises ${ }^{57}$. Comme on l'a remarqué auparavant, cette maison fut modifiée pour servir les besoins économiques des occupants. Or le propriétaire de la maison était Quintus Tullius, mais il est probable que ses affranchis géraient ses affaires en son absence. On peut donc supposer que la modification de la maison pour accueillir des activités économiques ait été faite pour mieux servir les activités - peut-être indépendantes - des affranchis de Quintus Tullius. On pourrait envisager, par exemple, que le rez-de-chaussée accueillait un lieu de stockage géré par Héracléon, un atelier géré par Alexandros et un logement à louer géré par Aristarchos, tandis que l'inscription bilingue soulignait leur aspiration sociale à s'intégrer dans la société délienne. De toute façon, la combinaison de l'étage luxueux et du rez-de-chaussée réservé aux activités économiques, servait les aspirations sociales des affranchis de Quintus Tullius.

Dans la salle (h), un graffito fait apparaître un cheval, un bateau et la phrase suivante :

Mṿฺọ [---]

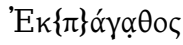

$\left[.{ }^{\text {ca 3-4. }}.\right]$ ПАГА $\Theta \Omega^{58}$.

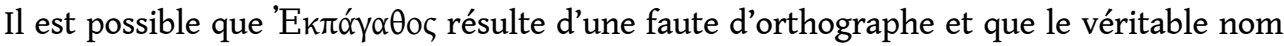

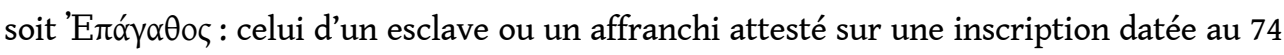

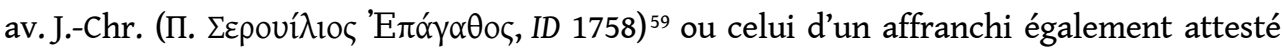
sur une inscription découverte dans l'extrémité sud du portique de Philippe (GD 3 ; ID

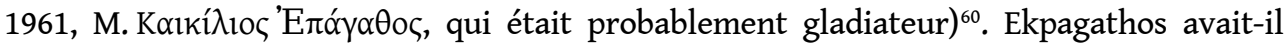
loué la pièce (h) ou travaillait-il avec les affranchis de Quintus Tullius? Il est intéressant de noter qu'une figurine d'Aphrodite orientale a été découverte dans cette même salle (inv. $n^{\circ} \mathrm{A} 2498$ ), ce qui tend à suggérer la pluralité d'identités des locataires, ainsi que l'importance des marchands italiens de Délos dans la propagation des cultes égyptiens en Campanie $^{61}$. Le graffito de bateau pourrait être associé aux cultes égyptiens et au rapport à la mer de la diaspora commerciale égyptienne, responsable de la propagation des cultes égyptiens de centres comme Délos jusqu'en Italie et en Occident ${ }^{62}$.

L'inscription provient de l'étage supérieur de la maison, probablement du balcon nord disposé autour de la cour (c): elle a été trouvée dans les couches supérieures des décombres de l'étage, à proximité du mur sud de la salle $(\mathrm{g})^{63}$. L'inscription et la statue qui l'accompagnait devaient pouvoir être vues des visiteurs de la maison, puisque les salles de réception étaient situées à cet étage. L'accès à l'étage se faisait par le vestibule (a), dans la partie sud de la cour. Les salles de réception étaient situées dans la zone septentrionale de l'étage, au-delà du balcon nord où l'inscription et la statue étaient probablement 
installées. En arrivant sur le balcon sud sis autour de la cour, un visiteur devait voir l'inscription et la statue du balcon nord, à travers l'ouverture de la cour.

En somme, l'inscription bilingue de la maison de Quintus Tullius souligne la relation privée entre des affranchis et leur patron. Les premiers géraient probablement les affaires déliennes du second et le monument épigraphique visait probablement à souligner leur identité sociale et économique, elle-même liée aux activités pratiquées au rez-de-chaussée. Cette volonté d'affichage est notable sur d'autres inscriptions provenant d'espaces domestiques : par exemple, des maisons du couple athénien, de Cléopâtre et Dioskourides, et de Spurius Stertinius (la maison qui était accessible à partir de la rue à l'est de peribolos oriental du sanctuaire d'Apollon [GD 82] $)^{64}$.

La nouvelle organisation des maisons a façonné spatialement l'identité du groupe ethnique des Italiens à Délos. Ils se nommaient 'P $\omega \mu \alpha \tilde{o}$ or sur les inscriptions pour favoriser leurs affaires: cette romanité servaient leurs intérêts professionnels. Ils aménagèrent leur maison avec les mêmes préoccupations en tête, pour tirer profit du dynamisme de l'île, et pour afficher une identité sociale, déterminée en grande partie par leurs activités économiques.

Les maisons des négociants italiens à Délos fournissent en fait un exemple précoce de combinaison de l'élégance et des activités économiques dans le tissu urbain, connue par ailleurs par des exemples campaniens antérieurs ${ }^{65}$. Les études récentes de Monika Trümper et Pavlos Karvonis ont démontré que l'architecture commerciale et celle des boutiques à Délos fournissent des parallèles aux développements qui ont eu lieu dans l'Italie républicaine. Par exemple, dans certaines maisons, des boutiques et des ateliers ont été murés et des étages rendus accessibles par une entrée séparée, pour être loués séparément ${ }^{66}$. Mon analyse montre que l'architecture des maisons de Délos fournit un autre parallèle pour l'économie urbaine sophistiquée, que nous connaissons si bien par les sites de Pompéi et d'Herculanum ${ }^{67}$.

En somme, on ne peut pas dire que la communauté des négociants italiens à Délos ait formé une véritable élite, à la manière des élites négociantes des grands ports du XVIII ${ }^{\mathrm{e}}$ siècle de notre ère ${ }^{68}$. Cependant, on note l'émergence d'un groupe aisé, qui - sans appartenir juridiquement à l'élite - se manifestait de manière comparable à elle, en public et en privé. L'étude récente d'Emmanuel Mayer, qui se concentre sur les villes d'Italie, a employé le terme "middle class» pour décrire de tels groupes ${ }^{69}$. L'interprétation proposée par Mayer a suscité des réactions vives ${ }^{70}$, à juste titre, à cause de l'anachronisme du terme "middle class ». Même si le terme qu'il a utilisé est problématique, il parait évident que la situation qu'il décrit n'est pas fictive. Je pense donc, qu'il faut trouver une autre façon de décrire nos négociants italiens.

BIBLIOGRAPHIE

Adams J. N. (2002), « Bilingualism at Delos », dans Adams J.N., Janse M. et Swain S. éd., Bilingualism in Ancient Society: Language Contact and the Written Text, Oxford, p. 103-127. 
- (2003), Bilingualism and the Latin language, New York.

Auda Y. et Boussac M.-Fr. (1996), « Étude statistique d'un dépôt d'archives à Délos », dans Boussac M.-Fr. et Invernizzi A. éd., Archives et sceaux du monde hellénistique. Actes du colloque de Turin 1993, Athènes, p. 511-522.

Barrett C. E. (2011), Egyptianizing Figurines from Delos: A Study in Hellenistic Religion, Leiden.

Basch L. (1973), « Graffites navals à Délos », Études déliennes publiées à l'occasion du centième anniversaire des fouilles de l'École française d'Athènes à Délos, BCH Suppl. 1, Paris, p. 65-76.

- (1987), Le musée imaginaire de la marine antique, Athènes.

- (1989), « Les graffiti de Délos », dans Tzalas H.E. éd., Tropisi. Proceedings of the 1st International Symposium on Ship Construction in Antiquity. Piraeus, 30 August-1 September 1985, Athènes, p. 17-23.

Baslez M.-F. (2002), « Mobilité et ouverture de la communauté “romaine” de Délos : amitiés, mariages mixtes, adoptions ", dans Müller Chr. et Hasenohr Cl. éd., Les Italiens dans le monde grec (II s. av. J.-C./I Ir s. ap. J.-C.) : circulation, activités, intégration. Actes de la table-ronde de l'École normale supérieure (Paris 14-16 mai 1998), BCH Suppl. 41, Athènes, p. 55-65.

Bezerra de Meneses U. et Sarian H. (1973), « Nouvelles peintures liturgiques de Délos », dans Études déliennes publiées à l'occasion du centième anniversaire des fouilles de l'École française d'Athènes à Délos, BCH Suppl. 1, Paris, p. 77-109.

Bonini P. (2006), La casa nella Grecia romana. Forme e funzioni dello spazio privato fra I e VI secolo, Antenor Quaderni 6, Rome.

Boussac M.-Fr. (1982), « À propos de quelques sceaux déliens », BCH 106, p. 427-446.

- (1988), « Sceaux Déliens », RA 2, p. 307-340.

- (1992), Les sceaux de Délos 1. Sceaux publics, Apollon, Hélios, Artémis, Hécate, Recherches francohelléniques 2 , Athènes-Paris.

- (1993), « Archives personnelles à Délos », CRAI 137, p. 677-693.

Boussac M.-F. et Moretti J.-C. (1995), « Revue de N. K. Rauh. The sacred bonds of commerce : religion, economy, and trade society at Hellenistic Roman Delos, $1993 »$, Topoi 5, 561-572.

Bruneau Ph. (1968), « Contribution à l'histoire urbaine de Délos à l'époque hellénistique et à l'époque impériale », $B C H$ 92, p. 633-709.

- (1970), Recherches sur les cultes de Délos à l'époque hellénistique et à l'époque impériale, Bibliothèque des écoles française d'Athènes et de Rome 217, Paris.

— (1975), « Deliaca », BCH 99, p. 267-311.

- (1978), « Deliaca (II) », BCH 102, p. 109-171.

- (1982), « Les Israélites de Délos et la juiverie délienne », BCH 56, p. 465-504.

- (1995), «La maison délienne », RAMAGE. Revue d'archéologie moderne et d'archéologie générale 12, p. 77-118.

Bruneau Ph. et Ducat J. (2005), Guide de Délos, Sites et Monuments 1, Athènes-Paris ( $4^{\mathrm{e}}$ éd. refondue et mise à jour).

Bruneau Ph., Vatin Cl., Bezerra de Meneses U., Donnay G., Lévy E., Bovon A., Siebert G., Grace V. R., Savvatianou-Pétropoulakou M., Lyding Will E. et Hackens T. (1970), L'îlot de la maison des comédiens, Exploration archéologique de Délos 27, Paris. 
Bizard L. (1907), « Descriptions des ruines (pl. XIV)», BCH 31, p. 471-503.

Brunet M. (1998), « L'artisanat dans la Délos hellénistique : essai de bilan archéologique », Topoi 8, p. 681-691.

Bulard M. (1908), Peintures murales et mosaïques de Délos, MMAI 14, Paris.

- (1926), Description des revêtements peints à sujets religieux, Exploration archéologique de Délos 9, Paris.

Chamonard J. (1922-1924), Le quartier du Théâtre : étude sur l'habitation délienne à l'époque hellénistique, Exploration archéologique de Délos 8, Paris.

Christol M. (2002), « Narbonne : un autre emporion à la fin de l'époque républicaine et à l'eepoque augustéenne » dans Müller Chr. et Hasenohr Cl. éd., Les Italiens dans le monde grec (II ${ }^{e}$ s. av. J.-C./I $I^{e r} s$. ap. J.-C.) : circulation, activités, intégration. Actes de la table-ronde de l'École normale supérieure (Paris 14-16 mai 1998), BCH Suppl. 41, Athènes, p. 41-54.

Cébeillac-Gervasoni M. (2002), « Note relative aux élites du Latium et de la Campanie et à leurs rapports avec la Méditerranée orientale ", dans Müller Chr. et Hasenohr Cl. éd., Les Italiens dans le monde grec (II ${ }^{e}$ s. av. J.-C./Irr s. ap. J.-C.) : circulation, activités, intégration. Actes de la table-ronde de l'École normale supérieure (Paris 14-16 mai 1998), BCH Suppl. 41, Athènes, p. 22-28.

Couilloud M.-Th. (1974), Les monuments funéraires de Rhénée, Exploration archéologique de Délos 30, Paris.

Deniaux É. (2002), « Les gentes de Délos et la mobilité sociale à Rome au I ${ }^{\mathrm{er}}$ s. av. J.-C. : l'exemple de Marcus Seius et des Seii », dans Müller Chr. et Hasenohr Cl. éd., Les Italiens dans le monde grec (II ${ }^{e} s$. av. J.-C./IIr s. ap. J.-C.) : circulation, activités, intégration. Actes de la table-ronde de l'École normale supérieure (Paris 14-16 mai 1998), BCH Suppl. 41, Athènes, p. 29-39.

Ferrary J.-L., Hasenohr Cl., Le Dinahet M.-Th. avec la collaboration de Boussac, M.-Fr. (2002), « Liste des Italiens de Délos », dans Müller Chr. et Hasenohr Cl. éd., Les Italiens dans le monde grec (II s. av. J.-C./I Ir s. ap. J.-C.) : circulation, activités, intégration. Actes de la table-ronde de l'École normale supérieure (Paris 14-16 mai 1998), BCH Suppl. 41, Athènes, p. 183-239.

Flohr M. (2007), « Nec quicquam ingenuum habere potest officina? », dans Spatial contexts of urban production at Pompeii, AD 79, BABesh 82 (1), p. 129-148.

Frier B.W. (1977), « The rental market in early imperial Rome », JRS 67, p. 27-37.

Gassner V. (1986), Die Kaufläden in Pompeii, Vienne.

Hallett Ch. H. (2001), The Roman Nude: Heroic Portrait Statuary, 200 BC-AD 300, Oxford.

Hasenohr Cl. (2001), « Les monuments des collèges italiens sur l'agora des Compétaliastes à Délos » dans Marc J.-Y. et Moretti J.-Ch. éd., Constructions publiques et programmes édilitaires en Grèce entre le II ${ }^{e}$ s. av. J.-C. et le I $I^{e r}$ s. ap. J.-C. Actes du colloque organisé par l'Ecole française d'Athènes et le CNRS (Athènes 14-17 mai 1995), Athènes-Paris, p. 329-348.

- (2002), « Les collèges de magistri et la communauté italienne de Délos » dans Müller Chr. and Hasenohr Cl. éd., Les Italiens dans le monde grec (II ${ }^{e}$ s. av. J.-C./Ir s. ap. J.-C.) : circulation, activités, intégration. Actes de la table-ronde de l'École normale supérieure (Paris 14-16 mai 1998)), BCH Suppl. 41, Athènes, p. 67-76.

- (2003), « Les Compitalia à Délos », BCH 127, p. 167-249.

- (2007), « Les Italiens à Délos : entre romanité et hellénisme », Pallas. Revue d'études antiques 73, p. 221-232. 
- (2008a), « Mercure à Délos » dans Bouet A. éd., D’Orient et d'Occident : Mélanges offerte à Pierre Aupert, Bordeaux, p. 27-38.

- (2008b), « Le bilinguisme dans les inscriptions des magistri de Délos » dans Biville Fr., Decourt J.-Cl. et Rougemont G. éd., Bilinguisme Gréco-Latin et épigraphie. Actes du colloque organisé à l'Université Lumière-Lyon 2 (17-19 mai 2004), Lyon, p. 55-70.

Hasenohr Cl. et Müller Chr. (2002), « Gentilices et circulation des Italiens : queques réflexions méthodologiques » dans Chr. Müller and Cl. Hasenohr éd., Les Italiens dans le monde grec (II ${ }^{e}$ s. av. J.C./Ir ${ }^{e r}$ ap. J.-C.) : circulation, activités, intégration. Actes de la table-ronde de l'École normale supérieure (Paris 14-16 mai 1998), BCH Suppl. 41, Athènes, p. 11-20.

Hatzfeld J. (1912), « Les Italiens résidents à Délos », BCH 36, p. 5-218.

- (1919), Les trafiquants italiens dans l'Orient hellénique, Paris.

Hermary A., Jockey Ph., Queyrel Fr., Marcadé J. et Collet Ph. (1996), Sculptures déliennes, École française d'Athènes. Sites et monuments 17 , Athènes-Paris.

Holleran Cl. (2012), Shopping in Ancient Rome: the Retail Trade in the Late Republic and the Principate, Oxford.

Husson G. (1983), Oikia : le vocabulaire de la maison privée en Égypte d'après les papyrus grecs, Paris.

Huzar E. G. (1962), « Roman-Egyptian relations in Delos », CJ 57, p. 169-178.

Karvonis P. (2008), « Les installations commerciales dans la ville de Délos à l'époque hellénistique », BCH 132, p. 153-219.

Kay Ph. (2014), Rome's economic revolution, Oxford Studies on the Roman Economy, Oxford.

Keay S. J. et Terrenato N. éd. (2001), Italy and the West: Comparative Issues in Romanization, Oxford. Kreeb M. (1988), Untersuchungen zur figürlichen Ausstattung Delischer Privathäuser, Chicago.

Langner M. (2001), Antike Graffitizeichnungen: Motive, Gestaltung und Bedeutung, Wiesbaden. Laumonier A. (1956), Les figurines de terre cuite, Exploration archéologique de Délos 23, Paris. Le Roux P. (2004), « La romanisation en question » Annales (HSS) $259^{\mathrm{e}}$ année, p. 287-311.

Lespagnol A. (1997), Messieurs de Saint-Malo : une élite négociante au temps de Louis XIV, Rennes.

Malaise M. (1972), Les conditions de pénétration et de diffusion des cultes égyptiens en Italie, Études préliminaires aux religions orientales dans l'Empire romain 22, Leiden.

Marcadé J. (1988), « Sur la sculpture hellénistique délienne », dans Akten des XIII. internationalen Kongresses für klassische Archäologie, Berlin 1988, Mainz am Rhein, p. 145-150.

Martzavou, P. (2010), « Les cultes isiaques et les Italiens entre Délos, Thessalonique et l'Eubée », Pallas. Revue d'études antiques 84, p. 181-205.

Mavrojannis T. (2002), « Italiens et orientaux à Délos : considérations sur l"'absence" des negotiatores romains dans la méditerranée orientale ", dans Müller Chr. et Hasenohr Cl. éd., Les Italiens dans le monde grec (II ${ }^{e}$ s. av. J.-C./I Irr s. ap. J.-C.) : circulation, activités, intégration. Actes de la table-ronde de l'École normale supérieure (Paris 14-16 mai 1998), BCH Suppl. 41, Athènes, p. 163-79.

Mayer E. (2012), The Ancient Middle Classes : Urban Life and Aesthetics in the Roman Empire, 100 BCE-250 CE, Cambridge MA.

McClain T. D. et Rauh N. K. (1996) « Signs of a Woman's Influence? The Dedications of the Stertinian Familia at Delos », Aevum 70, p. 47-68. 
Molinier S. (1914), Les « maisons sacrées » de Délos au temps de l'independance de l'île (315-166/5 av. J.C.), Études préliminaires aux religions orientales dans l’Empire romain 22, Paris.

Monteix N. (2010), Les lieux de métier : boutiques et ateliers d'Herculanum, Bibliothèque des écoles française d'Athènes et de Rome 344, Rome.

Müller Ch. et Hasenohr Cl. éd. (2002), Les Italiens dans le monde grec (II ${ }^{e}$ s. av. J.-C./I I s. ap. J.-C.) : circulation, activités, intégration. Actes de la table-ronde de l'École normale supérieure (Paris 14-16 mai 1998), BCH Suppl. 41, Athènes.

Nevett L. C. (2010), Domestic Space in Classical Antiquity, Cambridge.

Noreña C. (2013), « Revue de E. Mayer (2012), The Ancient Middle Classes : Urban Life and Aesthetics in the Roman Empire, 100 BCE-250 CE », AHR 118, p. 1576-1577.

Papaioannou M. (2002), Domestic architecture of Roman Greece, thèse de l'université de British Columbia, Vancouver.

- (2010), « The Evolution of the Atrium-house: A Cosmopolitan Dwelling in Roman Greece », dans Ladstätter S. et Scheibelreiter V. éd., Städtisches Wohnen im östlichen Mittelmeerraum. 4. Jh. v.-1.Jh. n. Chr. Akten des Kolloquiums von 24.-27. 10. 2007, Vienne, p. 81-115.

- (à paraître), « Roman Villas in Greece and the Islands », dans Métraux G.P.R. et Marzano A., Roman villas in the Mediterranean basin, Cambridge-New York.

Papageorgiou-Venetas A. (1981), Délos. Recherches urbaines sur une ville antique, Berlin.

Plassart A. (1916), « Fouilles de Délos, exécutées aux frais de M. le Duc de Loubat (1912-1913). Quartier des habitations privées à l'est du Stade (pl. V-VII)», BCH 40, p. 145-256.

Purcell N. (1995), « The Roman Villa and the Landscape of Production », dans Cornell T. J. et Lomas K. éd., Urban Society in Roman Italy, New York, p. 151-179.

Rauh N. K. (1993), The Sacred Bonds of Commerce: Religion, Economy, and Trade Society at Hellenistic Roman Delos, 166-87 B. C, Amsterdam.

Récit d'une aventure (1992), Récit d'une aventure : les graffiti marins de Délos: Musée d'histoire de Marseille, 18 décembre 1992-22 mars 1993, Marseille.

Reger G. (1994), Regionalism and Change in the Economy of Independent Delos, 314-167 B.C., Hellenistic culture and society 14 , Berkeley.

Rizakis A. (2002) «L'émigration romaine en Macédoine et la communauté marchande de Thessalonique : Perspectives économiques et sociales ", dans Müller Chr. et Hasenohr Cl. éd., Les Italiens dans le monde grec (II ${ }^{e}$ s. av. J.-C./IIr s. ap. J.-C.) : circulation, activités, intégration. Actes de la table-ronde de l'École normale supérieure (Paris 14-16 mai 1998), BCH Suppl. 41, Athènes, p. 109-132.

Rizakis A. D. et Touratsoglou I. P. éd. (2013), Farms and Villae Rusticae in Greece During the Roman Period, MELETEMATA 68, Athènes.

Roussel P. (1908), « Les Athéniens mentionnés dans les inscriptions de Délos (pl. IV) [Contribution à la prosopographia attica de J. Kirchner] », BCH 32, p. 303-444.

- (1931), « La population de Délos à la fin du $\mathrm{II}^{\mathrm{e}}$ siècle avant J.-C. », BCH 55, p. 438-449.

- (1987), Délos, colonie Athénienne, réimpression augmentée de compléments bibliographiques et des concordances épigraphiques par Philippe Bruneau, Marie-Thérèse Couilloud-Le Dinahet, Roland Étienne, Paris.

Roussel P. et Bizard L. (1907), « Fouilles de Délos, exécutées aux frais de M. le Duc de Loubat (1904). Inscriptions (suite) », BCH 31, p. 421-470. 
Roussel P. et Hatzfeld J (1910), « Fouilles de Délos exécutées aux frais de M. le Duc de Loubat. Décrets, dédicaces et inscriptions funéraires (1905-1908) II », BCH 34, p. 355-423.

Siebert G. (1969), « Délos. Report in Chronique des fouilles de l'École française en 1968 (ed. G. Daux) », BCH 93, p. 1031-1044.

- (2001), L'îlot des bijoux, l'îlot des bronzes, la Maison des sceaux, Exploration archéologique de Délos 38 , Athènes et Paris.

Solin H. (1982), « Appunti sull'onomastica romana a Delo » dans Coarelli F., Musti D. et Solin H. éd., Delo e l'Italia, Rome, p. 101-117.

Spawforth A. (2012), Greece and the Augustan Cultural Revolution, Cambridge-New York.

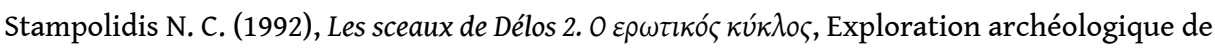
Délos 2A, Athènes-Paris.

Stek T. D. (2008), «A Roman cult in the Italian countryside? The Compitalia and the shrines of the Lares Compitales », BABesch 83, p. 111-132.

Stewart A. F. (1979), Attika: Studies in Athenian Sculpture of the Hellenistic Age, Londres.

Tang B. (2005), Delos, Carthage, Ampurias: The Housing of Three Mediterranean Trading Centres, Rome. Terrenato N. (2001), « Introduction », dans Keay S. J. et Terrenato N. éd., Italy and the West: Comparative Issues in Romanization, Oxford, p. 1-6.

Touloumakos J. (1995), « Bilingue [Griechisch-Lateinische] Weihinschriften der römischen Zeit », Tekmeria 1, p. 79-129.

Tran N. (2013), Dominus tabernae : le statut de travail des artisans et des commerçants de l'occident romain ( $I^{e r}$ s. av. J.-C.-III siècle ap. J.-C.), Bibliothèque des écoles française d'Athènes et de Rome 160 , Rome.

Tran Tam Tinh V. (1964), Essai sur le culte d'Isis à Pompéi, Paris.

Tréheux J. (1952), «Études d'épigraphie délienne », BCH 76, p. 562-595.

- (1992), Inscriptions de Délos, I. Les étrangers, à l'exclusion des Athéniens de la clérouchie et des Romains , Paris.

Trümper M. (1998), Wohnen in Delos : Eine baugeschichtliche Untersuchung zum Wandel der Wohnkulture in hellenistischer Zeit, Rahden.

- (2005), « Modest Housing in Late Hellenistic Delos », dans Ault B. A. et Nevett L. C. éd., Ancient Greek Houses and Households: Chronological, Regional, and Social Diversity, Philadelphia, p. 119-139.

- (2006), « Negotiating Religious and Ethnic Identity : The Case of Clubhouses in Late Hellenistic Delos », Hephaistos 24, p. 113-40.

- (2007), « Differentiation in the Hellenistic Houses of Delos: the Question of Functional Areas » dans Westgate R., Fisher N. et Whitley, J. éd., BUILDING COMMUNITIES : House, Settlement and Society in the Aegean and Beyond. Proceedings of a Conference Held at Cardiff University, 17-21 April 2001, Londres, p. 323-334.

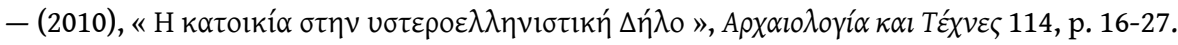

- (2011), « Where the Non-Delians Met in Delos: The Meeting-Places of Foreign Associations and Ethnic Communities in Late Hellenistic Delos ", dans van Nijf O. et Alston R. éd., Political Culture in the Greek City after the Classical Age, Leuven, p. 49-100. 
Vallois R. (1944), L'architecture hellénique et hellénistique à Délos jusqu'à l'éviction des Déliens (166 av. J.C.). v. 1. Les monuments, Bibliothèque des écoles française d'Athènes et de Rome 157, Paris.

Veyne P. (1999), «L'identité grecque devant Rome et l'empereur », REG 112, p. 510-567.

Vial Cl. (1984), Délos Indépendante, BCH Suppl. 10, Athènes-Paris.

Walbank F. W. (1979), The rise of the Roman Empire by Polybius, traduit par I. Scott-Kilvert, Harmondsworth-New York.

Wallace-Hadrill A. (1994), Houses and Society in Pompeii and Herculaneum, Princeton.

- (2008), Rome's Cultural Revolution, Cambridge.

Webster J. (2001) «Creolizing the Roman Provinces », AJA 105, p. 209-225.

Will E. (1951), « Le sanctuaire syrien de Délos », Annales archéologiques de Syrie 1, p. 59-79.

Wilson A. J. (1966), Emigration from Italy in the Republican age of Rome, Manchester.

Woolf G. (1998), Becoming Roman: The Origins of Provincial Civilization in Gaul, Cambridge-New York. - (2001), «The Roman Cultural Revolution in Gaul », dans Keay S. J. et Terrenato N. éd., Italy and the West: Comparative Issues in Romanization, Oxford, p. 173-186.

Wurmser H. (2008), Étude d'architecture domestique la maison en Grèce à l'époque impériale, thèse de l'université Paris-Sorbonne (Paris IV), Paris.

- (2010a), « Hellenistic living in the Aegean », dans Ladstätter S. et Scheibelreiter V. éd., Städtisches Wohnen im östlichen Mittelmeerraum. 4. Jh. v.-1.Jh. n. Chr. Akten des Kolloquiums von 24.-27. 10. 2007, Vienne, p. 13-25.

- (2010b), «L'habitat grec à l'époque impériale : traditions et nouveautés », Dossiers d'Archéologie 342 , p. 88-97.

Zarmakoupi M. (2013a), «The villa culture of Roman Greece », dans Rizakis A. D. et Touratsoglou I. P. éd., Farms and villae rusticae in Greece during the Roman period, MELETEMATA 68, Athènes, p. 742-751.

- (2013b), « The Quartier du Stade on late Hellenistic Delos: a Case Study of Rapid Urbanization (fieldwork seasons 2009-2010) », ISAW Papers 6. URL : http://dlib.nyu.edu/awdl/isaw/isawpapers/6/

- (2013c), « The City of Late Hellenistic Delos and the Integration of Economic Activities in the Domestic Sphere ", CHS Research Bulletin 1, 2. URL : http://nrs.harvard.edu/ urn-3:hlnc.essay:ZarmakoupiM.The_City_of_Late_Hellenistic_Delos.2013

- (2014), « Private Villas: Italy and the Provinces », dans Ulrich R. B. et Quenemoen C.K. éd., A companion to Roman Architecture, Malden-Oxford, p. 363-380.

- (2016), « The Spatial Environment of Inscriptions and Graffiti in Domestic Spaces: the Case of Delos », dans Benefiel R. et Keegan P. éd., Inscriptions in Private Spaces, Leiden, p. 50-79.

\section{NOTES}

1. Voir Polybe, XXX, 31, 10-12, et la discussion dans Walbank 1979, p. 459-60.

2. Voir Kay 2014, p. 202-206.

3. Voir Brunet 1998 ; Karvonis 2008. 
4. Roussel 1931; Tréheux 1952, p.582, n.3; Couilloud 1974, p.307-335. Pour les estimations quantitatives de la population délienne sous l'Indépendance: Vial 1985, p. 17-20 ; Bruneau 1970, p. 262-263 ; Reger 1994, p. 83-85.

5. Voir Treheux 1992.

6. Voir les listes des Italiens des Délos : Hatzfeld 1912 ; Ferrary et al. 2002. Voir également Wilson 1966, p. 99-119. Sur la population italienne de Délos voir les études publiées dans Müller et Hasenohr 2002 éd., particulièrement: Hasenohr et Müller 2002; CébeillacGervasoni 2002 ; Déniaux 2002 ; Christol 2002 ; Baslez 2002 ; Hasenohr 2002 ; Mavrojannis 2002. Voir également les articles de Claire Hasenohr sur l'Agora des Compétaliastes (Hasenohr 2001, 2003 et 2008a) et sur l'identité et des Italiens à Délos (Hasenohr 2007).

7. Bruneau 1968, p. 665-666; 1995, p. 106-108. Sur l'architecture de maisons déliennes voir les publications de quartiers résidentiels de Délos : Chamonard 1922-24; Bruneau et al. 1970; Plassart 1916; Siebert 2001. Voir également les synthèses récentes sur l'architecture de maisons déliennes: Trümper 1998, 2005, 2007 et 2010; Tang 2005; Nevett 2010, p. 63-88 (ch. 4, «Housing and cultural identity: Delos, between Greece and Rome »).

8. Keay et Terrenato éd. 2001 (particulièrement Terrenato 2001 et Woolf 2001) ; Woolf 1998; Webster 2001; Voir Le Roux 2004, sur le débat autour de la notion de « romanisation ». Voir également Wallace-Hadrill 2008, particulièrement p. 3-37, ch. 1, sur ce débat et les approches alternatives visant à comprendre les processus culturels désignés jusqu'à présent par ce terme ou par d'autres (e.g. hellénisation).

9. Voir Le Roux 2004, p. 307-311. Pour une discussion de ce processus vis-à-vis de la Grèce : Veyne 1999 ; Spawforth 2012, p. 1-58.

10. Wallace-Hadrill 2008, p. 14-28.

11. Sur la question de la forme de la maison grecque à l'époque de la domination romaine, voir : Bonini 2006 et les thèses de M. Papaioannou (2002 [inédit]) et de H. Wurmser (2008 [inédit]), ainsi que Papaioannou 2010, Wurmser 2010a et 2010b. Sur la question de la forme de villas romaines en Grèce voir Zarmakoupi 2013a et Papaioannou (à paraître), ainsi que les articles présentant les villae rusticae en Grèce dans Rizakis et Touratsoglou éd. 2013.

12. GD suivi d'un numéro renvoi à la présentation de chaque édifice dans la dernière édition du Guide de Délos (Bruneau et Ducat 2005).

13. Roussel 1908, p. 432, n 46 ; Roussel 1987, p. 38, nº 12 ; Kreeb 1988, p. 17-21, p. 282-284. En général : Chamonard 1922-24, p. $39 f$ et 218 ; Trümper 1998, p. 273-274.

14. Vallois 1944, p. 384 ; Will 1951, p. 61 ; Bruneau 1968, p. 665 ; Bruneau 1970, p. 473 et 642. En général : Chamonard 1922-1924, p. 27-29 ; Trümper 1998, p. 255-257.

15. Bulard 1908, p.193-194, p. 197-198; Bruneau 1968, p.666. Pour une approche générale : Chamonard 1922, p. 404-410 ; Trümper 1998, p. 196-198.

16. Bulard 1926 ; Bruneau 1970, p. 589-620 ; Bezerra de Meneses et Sarian 1973 ; Hasenohr 2003 (particulièrement p. 219-223) ; Stek 2008, p. 115-116. Voir également : Siebert 2001, p. 149-154.

17. Sur les associations religieuses à Délos: Bruneau 1970, p.585-589, p.621-638; Trümper 2006 et 2011.

18. Hatzfeld 1912 ; Solin 1982, p. 111-117 ; Ferrary et al 2002.

19. Hatzfeld 1919, particulièrement p. 238-236; Hasenohr 2007. 
20. Hasenohr 2007. Sur le bilinguisme à Délos: Adams 2002; Adams 2003, p. 642-686; Hasenohr 2008b.

21. Hasenohr 2007.

22. Bruneau et al. 1970 ; Siebert 2001.

23. Chamonard 1922-24, p. 168-176. Voir aussi Trümper 1998, p. 17-18.

24. Wurmser 2010a, p. 13-14.

25. Trümper 1998.

26. Nevett 2010, p. 63-88 (ch. 4).

27. Chamonard 1922-24, particulièrement p. 163-191 (ch. 6).

28. Bruneau 1995.

29. Le catalogue de Hasenohr (2003, p. 219-223) concerne 31 ensembles. Le catalogue de Bulard concerne 54 ensembles, dont 27 ne fournissent aucun reste de peintures. Huit nouveaux ensembles ont été découverts (Bezerra de Meneses, Sarian 1973) depuis le catalogue de Bulard. Hasenohr prend en compte ceux que l'emplacement ou l'iconographie permettent de mettre en relation avec les Compitalia, les ensembles $\mathrm{n}^{\circ} 1,2$, $3,4,9,10,11,12,14,17,18,20,22,23,24,25,27,55,57,58,59,60$ (2003, p. 198, n. 174). Parmi eux, 18 concernent des maisons.

30. Hasenohr 2007, p. 228-229.

31. Hasenohr 2001, 2003, 2007 et 2008.

32. Hasenohr 2007, p. 232.

33. Zarmakoupi $2013 \mathrm{~b}$ et $2013 \mathrm{c}$.

34. Siebert 2001, p. 85-98; Trümper 1998, p. 209-210.

35. Plassart 1916, p. 175-207 ; Trümper 1998, p. 218-220.

36. Plassart 1916, p. 207-228 ; Trümper 1998, p. 220-221.

37. Sur les sceaux : Boussac 1982, p. 427-446; Boussac 1988, p. 307-340; Boussac 1992 et 1993 ; Stampolidis 1992 ; Auda et Boussac 1996.

38. Sur les bustes: Marcadé 1988, p. 145-150 ; Hermary et al. 1996, p. 218-219; Stewart 1979, p. 71; Hallett 2001, p. 106-107. Rauh suppose que les bustes représentent les banquiers célèbres L. Aufidius Bassus et son fils (maior et minor) : Rauh 1993, p. 217-218 ; contra : Boussac et Moretti 1995.

39. Plassart 1916, p. 205-207 ; Rauh 1993, p. 198-200 ; Kreeb 1988, p. 169-170.

40. Voir Zarmakoupi $2013 \mathrm{~b}$.

41. Zarmakoupi 2013c.

42. Plassart 1916, p. 22 ; Zarmakoupi 2013 b.

43. Voir Zarmakoupi $2013 \mathrm{~b}$.

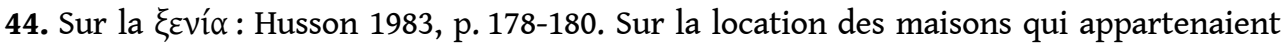
au sanctuaire d'Apollon à Délos: Molinier 1914. Pour une discussion des sources littéraires sur la location et sous-location d'une partie de la maison au début de l'Empire : Frier 1977, p. 27-37.

45. Chamonard 1922-24, p. 193-200.

46. Maison du lac (Bulard 1926, p. 56-61; Hasenohr 2003, n 1-2), Maison non fouillée à l'ouest de la Maison du lac (Bulard 1926, p. 62-63; Hasenohr 2003, n³), Maison au Nord 
de l'Établissement des Poseidoniastes de Bérytos (Bulard 1926, p. 64-69; Hasenohr 2003, n ○ 4), Maison II B du Quartier du théâtre (Bulard 1926, p. 86-87; Hasenohr 2003, n 11), Maison II D du Quartier du théâtre (Bulard 1926, p. 88-93 ; Hasenohr 2003, n 12), Maison III F du Quartier du théâtre (Bulard 1926, p. 94-97; Hasenohr 2003, n 13), Quartier du théâtre, Insula III, maison non fouillée à l'angle de la Rue 2 et de la ruelle $\zeta$ (Bulard 1926, p. 98-100; Hasenohr 2003, n 14), Maison VI G du Quartier du théâtre (Bulard 1926, p. 105-107; Hasenohr 2003, $\mathrm{n}^{\circ}$ 17), Maison VI H du Quartier du théâtre (Bulard 1926, p. 108-110; Hasenohr 2003, n 18), Maison non fouillée à l'Est de la Rue 2 du Quartier du théâtre (Bulard 1926, p. 112-114; Hasenohr 2003, n²0), Maison des dauphins (Bulard 1926, p. 115-116; Hasenohr 2003, n 22), Maison non fouillée à l'Ouest de la Maison des dauphins (Bulard 1926, p. 122-129; Hasenohr 2003, n 23), Maison IB du Quartier du stade (Bulard 1926, p. 130-132; Hasenohr 2003, n²4), Maison IC du Quartier du stade, porte ouvrant sur la rue Ouest (Bulard 1926, p. 133-149; Hasenohr 2003, n 25), Maison ID du Quartier du stade (Bulard 1926, p. 152-160 ; Hasenohr 2003, n² 27), Maison sise vis-à-vis de la Maison de la colline (Bezerra de Meneses et Sarian 1973; Bruneau 1970, p. 591-592; Bruneau 1975, p. 292 ; Hasenohr 2003, n 55), Habitation VI de l'îlot des bronzes (Siebert 1969, p. 1034-1035 ; Siebert 2001, p. 106, 152 ; Bruneau 1970, p. 591, III b ; Hasenohr 2003, n -58), Habitation II de l'îlot des bijoux (Siebert 2001, p. 152 ; Hasenohr 2003, n 59), Habitation III de l'îlot des bijoux (Siebert 2001, p. 152 ; Hasenohr 2003, n 60 ).

47. SEG 32 (1982) 812. Plassart 1916, p. 205-207 ; Bruneau 1982, p. 503, n. 90 ; Rauh 1993, p. 198-200 ; Kreeb 1988, p. 169-170.

48. Roussel et Bizard 1907, p. 459, fig. 16 ; Bizard 1907, p. 496; Bruneau 1970, p. 204, n 3 ; Kreeb 1988, p. 196-197. Pour les dédicaces de Stertinius à Delos, voir McClain et Rauh 1996. Pour une discussion de cette maison, voir Zarmakoupi (à paraître).

49. Voir Zarmakoupi 2014, p. 364-70.

50. Purcell 1995, p. 152.

51. Plassart 1916, p. 205-207 ; Rauh 1993, p. 198-200 ; Kreeb 1988, p. 169-170.

52. Ferrary et al. 2002, p. 218, $\mathrm{n}^{\circ} 4-7$.

53. Hatzfeld 1912, p. 86, Tullii, $n^{\circ} 2$; Ferrary et al. 2002, p. 218, $n^{\circ} 3$.

54. Plassart 1916, p. 206 ; Plassart 1916, p. 206 ; Hatzfeld 1912, p. 86, Tullii, nº 1 ; Ferrary et al. 2002 , p. $218, \mathrm{n}^{\circ} 8$.

55. Touloumakos 1995, p. 119-120 et 125 ; Adams 2002 ; Adams 2003, p. 642-662 ; Hasenohr 2007.

56. Touloumakos 1995, p. 90.

57. Adams 2002, particulièrement p. 115-119, p. 125-127. Voir également Hasenohr 2007 et $2008 \mathrm{~b}$.

58. Plassart 1916, p. 201. Sur les graffiti à Délos : Bruneau 1975, p. 286-289; Bruneau 1978, p. 146-151 (avec bibliographie antérieure); Basch 1973; Basch 1987, p.371-385 et p. 497-498; Basch 1989 ; Récit d'une aventure 1992. Pour une discussion sur la signification et fonction des graffiti: Langner 2001. Langner inclut les graffiti déliens dans sa discussion, mais pas ceux de cette maison.

59. Hatzfeld 1912, p. 77, Servilii n. 2 ; Ferrary et al. 2002, p. 214, Servilii n. 2.

60. Roussel and Hatzfeld 1910, p. 417, n' 81 ; Hatzfeld 1912, p. 22 ; Ferrary et al. 2002, p. 191, Caecilii n. 4. Bruneau (1995b, p. 48) remarque que cette inscription peut être datée à l'époque impériale. 
61. Laumonier 1956, p. 146, pl. 42, n 387 ; Barrett 2011, p. 335-336, p. 500-501, fig. F1, F2 et D19.

62. Huzar 1962, p. 173-174 ; Tran Tam Tinh 1964, p. 15-29 ; Malaise 1972, p. 268-311. Pour une discussion du caractère maritime de cultes égyptiens, voir : Rizakis 2002, p. 120-122 ; Martzavou 2010, p. 186-187. Pour une discussion sur les graffiti des maisons déliennes, voir Zarmakoupi (à paraitre).

63. Plassart 1916, p. 205-207 ; Kreeb 1988, p. 169.

64. Pour une discussion des inscriptions provenant des maisons déliens, voir Zarmakoupi 2016, p. 52-60.

65. Voir Mayer 2012, p. 25-34.

66. Trümper 2005 ; Karvonis 2008. Voir aussi : Mayer 2012, p. 34-41.

67. Voir Gassner 1986 ; Wallace-Hadrill 1994, p. 118-142 ; Flohr 2007, p. 136-141 ; Monteix 2010 ; Tran 2013, p. 339-356. Voir aussi : Holleran 2012, p. 100-105.

68. Voir par exemple les marchands du port de Saint-Malo : Lespagnol 1997, p. 71-117, p. 733-786.

69. Mayer 2012.

70. Voir par exemple Noreña 2013.

\section{RÉSUMÉS}

Cet article traite de l'architecture des maisons des négociants italiens à Délos pendant l'époque hellénistique et éclaire la structuration de leur espace domestique. En analysant l'organisation des maisons des négociants italiens mon but est d'aborder la façon avec laquelle l'espace domestique a été modelé afin de satisfaire les besoins diversifiés de ses habitants, ainsi que répondre à leurs aspirations sociales.

This article discusses the architecture of the houses of Italian merchants on Delos during the Hellenistic period and sheds light on the structuring of their domestic space. By analysing the organization of the houses of Italian merchants my goal is to address the ways in which domestic space was shaped so as to meet the diverse needs of the house owners as well as their social aspirations.

\section{INDEX}

Mots-clés : Délos, négociants italiens, maison délienne, espace domestique

Keywords : Italian merchants, Delian house, domestic space 


\section{AUTEUR}

MANTHA ZARMAKOUPI

Université de Birmingham 